\title{
DNA integrity is a critical molecular indicator for the assessment of male infertility
}

\author{
HUDA MOSSA OMRAN ${ }^{1}$, MOIZ BAKHIET ${ }^{2}$ and MARIAM GHLOOM DASHTI ${ }^{2}$ \\ ${ }^{1}$ College of Higher Education, Arabian Gulf University, Manama; \\ ${ }^{2}$ Department of Molecular Medicine, Princess Al-Jawhara Center for Genetics and Inherited Diseases, \\ College of Medicine and Medical Sciences, Arabian Gulf University, Bahrain
}

Received December 1, 2012; Accepted February 12, 2013

DOI: $10.3892 / \mathrm{mmr} .2013 .1390$

\begin{abstract}
Traditional semen analysis is routinely used for the assessment of male infertility. The present study aimed to evaluate the application of molecular techniques in determining the DNA integrity of the spermatozoa of infertile men in comparison to conventional analysis. Semen samples from 52 infertile patients were analyzed by conventional assays, flow cytometry (FCM) analysis for sperm DNA integrity and colorimetric assays for total antioxidant capacity (TAC). The traditional semen analyses showed that $48.1 \%$ of the patients had normospermic samples. The figure decreased to only $25 \%$ following the application of molecular tests. DNA FCM revealed that $67.3 \%$ of the patients had an abnormal chromatin status $(n=36)$, while at the same time, $36 \%$ showed aneuploidy features $(n=19)$, and $59.6 \%$ had an abnormal TAC level $(n=31)$. The normozoospermic samples had less DNA fragmentation and a higher mean seminal plasma TAC level $(1,500.92 \pm 280.29 \mu \mathrm{M})$ than the abnormal samples $(899.59 \pm 375.67 \mu \mathrm{M})$. Significant correlations were noted between chromatin condensation and the sperm count $(\mathrm{r}=587, \mathrm{P}<0.001)$, motility $(\mathrm{r}=0.625, \mathrm{P}<0.001)$, normal morphology $(\mathrm{r}=0.658, \mathrm{P}<0.001)$ and white blood cell $(\mathrm{WBC})$ count $(\mathrm{r}=-0.299, \mathrm{P}<0.005)$. In addition, the infertile subjects with abnormal chromatin condensation had significantly lower TAC levels than the other subjects and the level was markedly decreased in the samples with severe abnormalities (class 7 and 8). The presence of the aneuploidy cells was negatively correlated with the sperm concentration $(\mathrm{r}=-0.339, \mathrm{P}<0.005)$. In conclusion, traditional semen analysis has limited value in the prediction of male fertility and should be combined with other molecular tests to increase its sensitivity.
\end{abstract}

Correspondence to: Professor Moiz Bakhiet, Department of Molecular Medicine, Princess Al-Jawhara Center for Genetics and Inherited Diseases, P.O. Box 26671, Bahrain

E-mail:moiz@agu.edu.bh

Key words: oxidative stress, aneuploidy, semen analysis, sperm DNA

\section{Introduction}

Infertility is a common health problem that affects $15 \%$ of couples worldwide (1). Male partners may be involved in the etiology of half of the cases, either as the primary or secondary cause (2). There are numerous known causes for male infertility, however, important factors have been identified recently, including oxidative stress (OS), DNA fragmentation and aneuploidy of the sperm. OS is a product of the inequity between reactive oxygen species (ROS) and antioxidants in the body. It is a potent mechanism that leads to sperm damage and male infertility (3). Normally, the seminal plasma contains a specialized antioxidant system that provides effective protection against OS (4). A number of clinical studies have demonstrated a correlation between male infertility and OS (5-8). However, a fertility parameter that is not routinely evaluated is DNA integrity. Sperm chromatin is an extremely organized structure that consists of DNA and heterogeneous nucleoproteins. The predominate nucleoproteins in the mature sperm are the protamines, which replace the histones during spermiogenesis (9). Normal chromatin is essential for the transmission of paternal genomic information and it is well documented that fertility is negatively correlated with defective sperm chromatin or DNA fragmentation $(10,11)$.

Apart from DNA fragmentation, aneuploidy is another form of abnormality in sperm DNA. Human spermatozoa are haploid cells $(n=23)$ that contain 22 autosomes and one sex chromosome, either X or Y. Aneuploidy is the condition in which the sperm has an extra chromosome (disomy) or a missing chromosome (nullisomy) (12). The present study aimed to achieve proper characterization of human semen, with particular interest in the sperm DNA, together with an evaluation of the oxidative status as a possible etiological factor for impaired fertility.

\section{Materials and methods}

Study population and sample collection. The present study was approved by the ethical review board of the Arabian Gulf University. Verbal and written consents were obtained from the volunteers. All patients received an explanation of the study prior to obtaining informed consent. Semen samples were collected from infertile male patients attending the Salmania 
Medical Complex. All samples were collected by masturbation into sterile containers following 3-4 days of sexual abstinence. Subsequent to liquification, a conventional analysis was performed according to the World Health Organization guidelines (WHO Laboratory Manual for the Examination of Human Semen and Sperm-Cervical Mucus Interaction, 1999). The variables taken into account were the volume of the ejaculate $(\mathrm{ml})$, the concentration of round cells $\left(\mathrm{x} 10^{6} / \mathrm{ml}\right)$, the sperm concentration $\left(\mathrm{x} 10^{6} / \mathrm{ml}\right)$, the forward motility $(\%)$ and the morphology (\% of normal forms). A leukocyte count (x10\% $\mathrm{ml}$ ) was carried out using a standard peroxidase test. All the semen samples were analyzed with flow cytometry (FCM) and the colorimetric assay for total antioxidant capacity (TAC).

\section{FCM analysis}

Sample preparation. Native sampled preparations of a single cell suspension were performed according to the method used by Ehemann et al (13). The semen samples were treated with two types of solutions; ethanol fixation and citric acid preparation.

Sample staining for sperm DNA analysis by FCM. The fixed samples were brought to room temperature prior to staining. Each type of fixed cell had a specific staining technique: The ethanol fixed cells were centrifuged at $337 \mathrm{x}$ g for $10 \mathrm{~min}$ and resuspended in $3 \mathrm{ml}$ 4',6-diamidino-2-phenylindole sulforhodamine (DAPI-SR) 101 staining solution. This was kept in the dark for $20 \mathrm{~min}$ at room temperature. The citric acid prepared cells were diluted with $3.5 \mathrm{ml}$ of DAPI-phosphate staining solution and directly analyzed.

Cytometry analysis of the fluorescence-stained fixed samples. FCM analyses were performed using a CyFLOW space flow cytometer (Partec, Münster, Germany) equipped with a UV laser diode and a $488 \mathrm{~nm}$ argon laser and filter combination for DAPI-stained single cells. The multicycle program was used for the histogram analysis; each histogram represented $3 \times 10^{4}$ cells for measuring the DNA index. Human lymphocyte nuclei from healthy donors were used as the internal standard for the calibration of the diploid DNA-index.

Analysis of the frequency histograms. The DNA frequency histograms were evaluated using cumulative frequency distributions ascribed to Hacker-Klom et al (14). The term CC was used to describe the condensed chromatin and indicate the haploid spermatozoa, which had a normal DNA content. Five categories were recognized in each histogram: Cells with a sub-haploid DNA content $<1 C C$ (debris that may be of apoptotic origin); mature haploid spermatozoa in the $1 \mathrm{CC}$ peak, haploid round spermatids in the $1 \mathrm{C}$ peak; diploid spermatozoa in the $2 \mathrm{CC}$ peak, cells registered to the right of the $2 \mathrm{CC}$ level, including $2 \mathrm{C}$ cells (leukocytes, $\mathrm{G}_{1}$-spermatogonia and primary spermatocytes at preleptotene), and to the right of $1 \mathrm{CC}$, which are cells in the DNA synthesis phase (S); and 4C cells (primary spermatocytes). The presence of aneuploidy cells was marked as another bivarient histogram with blue coloration. The software analyzed the two types of population cells in the same diagram.

\section{Semen TAC assay}

Sample preparation. The samples were prepared according to the method used by Said et al (15). Following liquefaction, aliquots $(300 \mu \mathrm{l})$ of each sample were centrifuged at $300 \mathrm{x} \mathrm{g}$ for $7 \mathrm{~min}$. The supernatant was aspirated and recentrifuged at
$300 \mathrm{x}$ g for another $10 \mathrm{~min}$. The seminal plasma was frozen at $-70^{\circ} \mathrm{C}$ until further use.

Colorimetric assay for TAC. The colorimetric assay for TAC was performed as per the method of Mahfouz et al (4). Frozen seminal plasma was thawed by placing the vials in an incubator at $37^{\circ} \mathrm{C}$ for $20 \mathrm{~min}$ and then immediately assessed for the TAC. The TAC of the seminal plasma was measured with the colorimetric method using the Cayman's Antioxidant Assay kit (Cayman's Chemicals Company, Ann Arbor, MI, USA). The seminal plasma samples were diluted at 1:10 with the assay buffer prior to assaying. All the reagents and samples were equilibrated to room temperature prior to beginning the assay. The samples and Trolox standards were assayed twice by two observers. The Trolox standards and reagent were prepared as per the manufacturer's instructions at the time of the assay. Trolox standard $(10 \mu \mathrm{l})$ and the samples were loaded into the corresponding wells of a 96-well plate. Then, $10 \mu \mathrm{l}$ metmyoglobin and $150 \mu \mathrm{l}$ chromogen were added to all the standard/sample wells. The reaction was initiated by adding $40 \mu l$ hydrogen peroxide immediately. The plate was covered and incubated for $5 \mathrm{~min}$ on a shaker at room temperature. Absorbance was monitored at $750 \mathrm{~nm}$ using an ELx800 Absorbance Microplate Reader (Thomas Scientific, Swedesboro, NJ, USA). The accuracy and sensitivity of the TAC assay kit were calculated according to the manufacturer's instructions.

Statistical analysis. Data analysis was performed using the SPSS 16 for Windows Software Package (SPSS Inc., Chicago, IL, USA). Data were expressed as percentages and mean \pm SD. To identify the independent association between the various seminal parameters and selected variables, a logistic regression analysis was carried out. $\mathrm{P}<0.05$ was considered to indicate a statistically significant difference.

\section{Results}

Study population and sample collection. The age range of the patients was 21-52 years, with a mean age of 30.8 \pm 6.7 years. In total, $86.5 \%$ of the patients presented with primary infertility, while $13.5 \%$ complained of secondary infertility. Based on the WHO criteria, patient samples were either normal or abnormal; $51.9 \%$ of the samples were abnormal and the rest were normozoospermic. As shown in Table I, the normozoospermic group had significantly higher sperm concentrations, motility and normal forms than the abnormal group. Contrary to expectation, not all of the normozoospermic samples had normal cytometry DNA analyses. Of 52 samples obtained from the infertile male patients, 36 semen samples demonstrated abnormal chromatin at an overall percentage of 67.3 , whereas 16 had normal chromatin at an overall percentage of 32.7 .

The samples were further divided into eight classes based on the classification described by Haker-Klom et al (14). A total of 8 samples (15.4\%) were classified as class 1 and all these samples where normozoospermic. Class 2 consisted of 9 samples (17.3\%), from which only one ejaculate had an abnormal spermiogram (leukocytospermia). Class 3 contained the histograms of 7 samples (13.5\%) and among them, only one ejaculate exhibited asthenoteratospermic features. Class 4 included two ejaculatory samples $(3.8 \%)$ that had abnormal spermiograms (oligoasthenoteratozoospermia and terato- 
Table I. Analysis of the standard seminal parameters in the normozoospermic and abnormal semen samples (mean \pm SD).

\begin{tabular}{lccr}
\hline Parameter & Normal & Abnormal & P-value \\
\hline Concentration $\left(\mathrm{x} 10^{6} / \mathrm{ml}\right)$ & $54.28 \pm 1.47$ & $15.41 \pm 1.66$ & $<0.005$ \\
Motility $\%$ grade A) & $64.12 \pm 8.60$ & $16.81 \pm 20.47$ & $<0.005$ \\
Vitality $(\%$ alive) & $76.84 \pm 8.61$ & $25.00 \pm 29.30$ & $<0.005$ \\
Morphology $(\%$ normal form) & $63.08 \pm 9.3$ & $15.65 \pm 20.07$ & $<0.005$ \\
Leukocyte count & $0.36 \pm 0.49$ & $3.22 \pm 3.46$ & $<0.005$ \\
\hline
\end{tabular}

Table II. Correlation between DNA integrity and conventional semen parameters.

\begin{tabular}{lccccc}
\hline & \multicolumn{2}{c}{ Correlation with chromatin condensation } & & \multicolumn{2}{c}{ Correlation with presence of aneuploidy cells } \\
\cline { 2 - 3 } \cline { 5 - 6 } Parameter & $\mathrm{r}$ & P-value & & $\mathrm{r}$ & P-value \\
\hline Concentration & 0.587 & $<0.001$ & & -0.339 & $<0.005$ \\
Normal morphology $\%$ & 0.658 & $<0.001$ & & 0.245 & NS \\
Motility & 0.625 & $<0.001$ & & 0.260 & NS \\
WBC count & -0.299 & $<0.005$ & & -0.039 & NS \\
\hline
\end{tabular}

NS, non-significant; WBC, white blood cell.

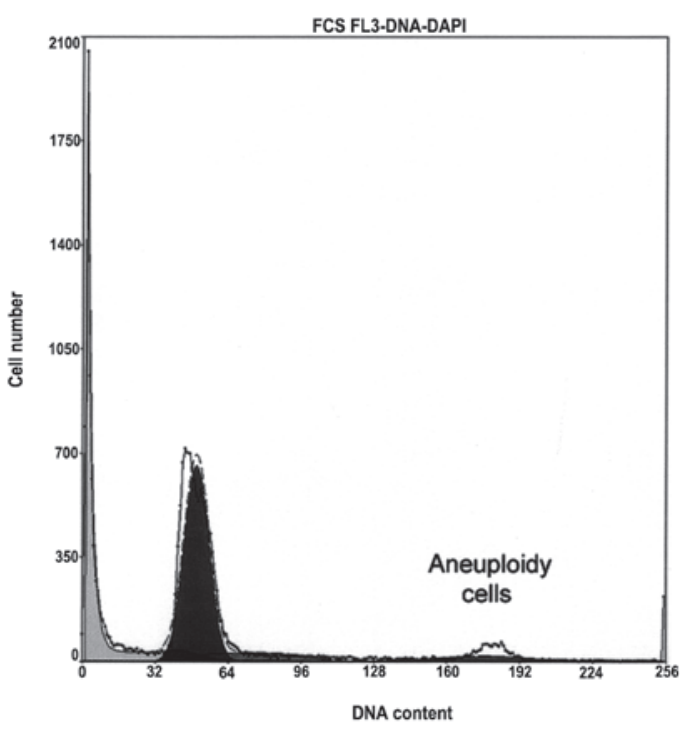

Figure 1. Cytometry analysis showing the concurrent presence of normal sperm chromatin condensation with the existence of aneuploidy cells (12.8\%). DAPI, 4',6-diamidino-2-phenylindole.

zoospermia). Class 5 contained the histograms of 4 samples (7.7\%) and only one ejaculate was normozoospermic. Class 6 contained 9 histograms (17.3\%) and barely two samples had normal spermiograms. Classes 7 and 8 had samples with abnormal spermiograms. Class 7 contained 6 samples $(11.5 \%)$, whose spermiograms showed severe oligozoospermia, while class 8 contained 7 azoospermic samples (13.5\%). The association between the parameters from the FCM analysis and the conventional semen parameters is described in Table II.

Aneuploidy. There were significant correlations between the sperm chromatin condensation and the sperm count,

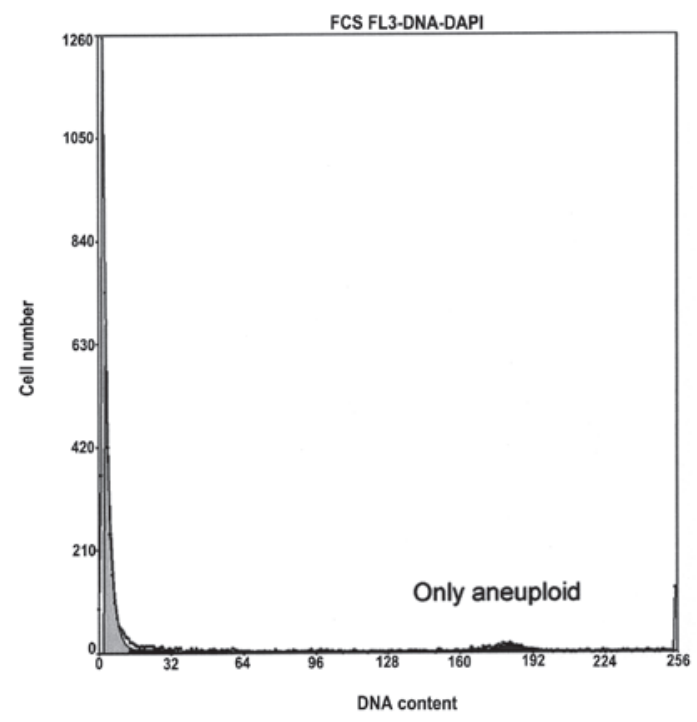

Figure 2. Cytometry analysis showing the absence of sperm with the existence of aneuploidy cells (60.24\%). DAPI, 4',6-diamidino-2-phenylindole.

morphology and motility; yet a significant but low negative correlation was observed between the chromatin condensation and the white blood cell (WBC) count. Another notable abnormality also observed during FCM analysis was the presence of aneuploidy cells. Of a total of 52 samples, 19 semen samples (36.54\%) showed aneuploidy features. The mean percentage of aneuploidy cells was higher in the samples with severe chromatin condensation abnormalities, for example, in classes 7 and 8, compared with other samples (Table III). These abnormal cells were present even in the normozoospermic samples (Table III). A noteworthy finding was the presence of 
Table III. Aneuploidy rate in relation to cytometry class in the 19 samples which had abnormal aneuploidy rates. ${ }^{\text {a }}$

\begin{tabular}{lccl}
\hline $\begin{array}{l}\text { Sample } \\
\text { No. }\end{array}$ & Aneuploidy, $\%$ & Class & \multicolumn{1}{c}{ Comment } \\
\hline 1 & 12.8 & 3 & Abnormal by WHO \\
2 & 60 & 8 & Aspermia \\
3 & 20.5 & 7 & Abnormal by WHO \\
4 & 14.17 & 6 & Abnormal by WHO \\
5 & 6.8 & 3 & Normal by WHO \\
6 & 31.93 & 6 & Abnormal by WHO \\
7 & 10.84 & 7 & Abnormal by WHO \\
8 & 14.59 & 3 & Normal by WHO \\
9 & 19.78 & 7 & Abnormal by WHO \\
10 & 28.4 & 7 & Abnormal by WHO \\
11 & 39.65 & 8 & Azoospermia \\
12 & 51.31 & 8 & Azoospermia \\
13 & 11.44 & 4 & Abnormal by WHO \\
14 & 11.2 & 3 & Normal by WHO \\
15 & 51.85 & 6 & Abnormal by WHO \\
16 & 38.37 & 6 & Abnormal by WHO \\
17 & 10 & 2 & Normal CC by WHO \\
18 & 48.9 & 8 & Azoospermia \\
19 & 8.9 & 5 & Abnormal by WHO \\
\hline & & &
\end{tabular}

${ }^{a}$ The remaining 33 samples were not included as all were devoid of such abnormalities. WHO, World Heralth Organization; CC, condensed chromatin.

aneuploidy cells in the semen samples with normal chromatin condensation (Fig. 1) and even in the samples with complete azoospermia (class 8; Fig. 2). There was a significant negative correlation between the sperm concentration and the presence of the aneuploidy cells. The samples with oligozoospermia were more likely to have an abnormal aneuploidy rate than the other samples (Table III). However, no significant correlation existed between the occurrences of these abnormal cells and the other semen parameters (Table II).

Concerning the TAC measurement, only $40.38 \%$ of the patients had a normal level. The normozoospermic samples had a higher mean level of TAC $(1,500.92 \pm 280.29 \mu \mathrm{M})$ than the abnormal samples $(899.59 \pm 375.67 \mu \mathrm{M})(\mathrm{P}<0.005)$. Moreover, the TAC levels were much lower in the samples with leukocytospermia than the other samples $(1050.40 \pm 495.33 \mu \mathrm{M}$ vs. $1,282.29 \pm 394.89 \mu \mathrm{M} ; \mathrm{P}<0.005)$. Another interesting feature was that the infertile subjects with an abnormal chromatin condensation had significantly lower TAC levels than the other subjects and that the level was markedly decreased in the samples with severe abnormalities (class 7 and 8). For class 1 the mean level of TAC was $1,715.25 \pm 321.43 \mu \mathrm{M}$, while the mean level of TAC in classes 7 and 8 was $769.83 \pm 241.81$ and 842.29 $\pm 415.09 \mu \mathrm{M}$, respectively.

\section{Discussion}

In clinical practice, an unsatisfactory diagnostic strategy remains for male infertility. This is due to the fact that despite the variety of etiological factors, the traditional technique for assessing human semen maintains its key role in the evaluation of male fertility (16). Traditional analysis provides a descriptive assessment of semen parameters, however, the normal values of these factors do not truly reflect the fertilization capacity of the sperm $(17,18)$. A previous study indicated the limited value of traditional analysis, particularly in assisted reproductive technology (ART) (8).

In the present study, traditional analysis revealed that $48.1 \%$ of the study group were normozoospermic and that they had higher parameter values than the abnormal samples, with the exception of the leukocyte content, as shown in Table I. Fertility workers depend on these results to differentiate between fertile and infertile males. However, this appeared to be an incorrect method of judgment when comparing the results of semen testing according to the WHO criteria and analyzing the other recent fertility parameters of the present study. Sperm DNA integrity has been recognized as an important parameter for semen quality (19), although it is not assessed routinely. A variety of assays have been developed to measure sperm DNA damage. Certain techniques detect the actual breaks in the DNA strands, whereas other approaches assess the vulnerability of the DNA to denaturation (20). FCM is a new method that has the ability to discriminate between the variations in DNA damage in the sperm (21). The principles of FCM are based on staining the sperm nuclei with a DNA stain to evaluate the ratio of single- and double-stranded DNA (following acid exposure that causes denaturation of the double-stranded DNA in the sperm with abnormal chromatin) (13). This technique assesses the sperm chromatin condensation and scores it according to the Hacker-Klom scoring system (14). Briefly, the Hacker-Klom scoring system divides the chromatin condensation state into eight classes, considering only classes 1 and 2 as normal. The present study revealed that $67.3 \%$ of the samples had abnormal chromatin. A number of these samples had normal class 1 chromatin and they represented $15.4 \%$ of the samples. Other samples had severe abnormalities and typical features of class 8 chromatin. The rest of the samples exhibited the features of the other classes (Fig. 2). The DNA fragmentation was greater in the males with abnormal semen parameters. The semen samples with a low sperm concentration, low motility and poor morphology exhibited more DNA fragmentation than the normal samples (Table II). These results were supported by several previously published studies $(8,22)$ that revealed an increase in the number of DNA abnormalities in the semen when there was an increase in the number of semen abnormalities. Another fertility parameter assessed by FCM was the aneuploidy rate of the spermatozoa. Aneuploidy gametes are a major cause of pregnancy loss, aneuploid births and developmental defects (23). In the present study, measurements of the aneuploidy rate demonstrated that $36.53 \%$ of the samples had abnormal aneuploidy cells. These abnormal cells mostly appeared in the samples with abnormal chromatin condensation (Table III). Nevertheless, the present study has also shown that these cells may be detected in samples with normal chromatin condensation (Fig. 1) and even in azoospermic samples (Fig. 2). A previous study detected abnormal aneuploidy cells in azoospermic samples (24), however, no study has recorded a high percentage of these cells in samples with normal chromatin condensation. The present study has demonstrated a negative 
correlation between the sperm count and the aneuploidy rate $(\mathrm{r}=-0.339, \mathrm{P}<0.005)$, a result that is in agreement with that of other studies that considered the patients with oligozoospermia at high risk of carrying chromosomal abnormalities, particularly if they chose to undergo the intracytoplasmic sperm injection (ICSI) program $(25,26)$.

The seminal TAC level was also assessed in the present study. A low seminal TAC level is frequently associated with male infertility (6). The present study has shown that $59.62 \%$ of the samples had an abnormal seminal TAC level; the seminal TAC was higher in the normozoospermic samples. Additionally, the TAC level was negatively correlated with leukocytospermia. This finding may be explained by the fact that the excess WBCs generated more ROS. These results are in concordance with the study by Sharma et al (27), which concluded that the presence of WBCs in semen samples is associated with OS and impaired fertility. Furthermore, there was a significant correlation between the sperm chromatin fragmentation and oxidative stress of the semen. The samples with a high degree of sperm DNA fragmentation had a low seminal TAC level. This agrees with previous studies that observed a negative correlation between the seminal TAC level and sperm chromatin fragmentation $(2,28)$. In the present study, a significant difference in the results of the various cellular and molecular tests compared with the classical semen analysis was observed. The semen analysis revealed that $48.1 \%$ of the study group had normal semen parameters, while by contrast, the aneuploidy rate measurements demonstrated that $63.47 \%$ of the samples were normal. The results of the chromatin condensation analysis showed that $32.7 \%$ of the samples were normal. Additionally, the measurement of the seminal TAC level revealed that only $40.38 \%$ of the samples were normal. Another finding of the present study was that only $25 \%$ of the patients had a normal spermiogram combined with normal chromatin condensation, a normal aneuploidy rate and a normal level of TAC. Only $23 \%$ of the patients had abnormal ejaculates by all analytical measurements. These variations in the results agreed with the study by Aitikan et al which considered that the semen parameters measured by conventional analysis are not able to assist the development of a diagnosis but may only provide a description of the seminal phenotype (17).

\section{References}

1. Sharma RS, Gaur KK, Pal PC, Manocha M, Tomar D, Khan AA et al: Semen characteristics: Advancement in andrological assessment. Indian J Clin Biochem 20: 173-183, 2005.

2. Tremellen K: Oxidative stress and male infertility - a clinical perspective. Hum Reprod Update 14: 243-258, 2008.

3. Makker K, Agarwal A and Sharma R: Oxidative stress and male infertility. Indian J Med Res 129: 357-367, 2009.

4. Mahfouz R, Sharma R, Lackner J, Aziz N and Agarwal A: Evaluation of chemiluminescence and flow cytometry as tools in assessing production of hydrogen peroxide and superoxide anion in human spermatozoa. Fertil Steril 92: 819-827, 2009.

5. Eskenazi B, Kidd SA, Marks AR, Sloter E, Block G and Wyrobek AJ: Antioxidant intake is associated with semen quality in healthy men. Hum Reprod 20: 1006-1012, 2005.

6. Agarwal A, Sharma RK, Nallella KP, Thomas AJ Jr, Alvarez Jg and Sikka SC: Reactive oxygen species as an independent marker of male factor infertility. Fertil Steril 86: 878-885, 2006.

7. Pasqualotto FF, Sundaram A, Sharma RK, Borges E Jr, Pasqualotto EB and Agarwal A: Semen quality and oxidative stress scores in fertile and infertile patients with varicocele. Fertil Steril 89: 602-607, 2008.
8. Varghese AC, Bragais FM, Mukhopadahyay D, Kundu S, Pal M, Bhattacharyya AK and Agarwal A: Human sperm DNA integrity in normal and abnormal semen samples and its correlation with sperm characterstics. Andrologia 41: 207-215, 2009.

9. Carrell DT, Emery BR and Hammoud S: Altered protamine expression and diminished spermatogenesis: what is the link? Hum Reprod Update 13: 313-327, 2007.

10. Evenson DP and Wixon R: Clincal aspects of sperm DNA fragmentation detection and male infertility. Theriogenology 65 : 979-991, 2006.

11. Bungum M, Humaidan P, Axmon A, Spano M, Bungum L, Erenpreiss J and Giwercman A: Sperm DNA integrity assessment in prediction of assisted reproduction technology outcome. Hum Reprod 22: 174-179, 2007.

12. Prisant N, Escalier D, Soufir JC, Morillon M, Schoevaert D, Misrahi $\mathrm{M}$ and Tachdjian G: Ultrastructural nuclear defects and increased chromosome aneuploidies in spermatozoa with elongated heads. Hum Reprod 22: 1052-1059, 2007.

13. Ehemann V, Sykora J, Vera-Delgado J, Lange A and Otto HF: Flow cytometric detection of spontaneous apoptosis in human breast cancer using the TUNEL-technique. Cancer Lett 194: 125-131, 2003

14. Hacker-Klom UB, Göhde W, Nieschlag E and Behre HM: DNA flow cytometry of human semen. Hum Reprod 14: 2506-2512, 1999.

15. Said TM, Kattal N, Sharma RK, Sikka SC, Thomas AJ Jr, Mascha E and Agarwal A: Enhanced chemiluminescence assay vs colorimetric assay for measurement of the total antioxidant capacity of human seminal plasma. J Androl 24: 676-680, 2003

16. Aziz N and Agarwal A: Evaluation of sperm damage: beyond the World Health Organization criteria. Fertil Steril 90: 484-485, 2008.

17. Aitken RJ, Baker MA and O'Bryan M: Shedding light on chemiluminescence:the application of chemiluminescence in diagnostic andrology. J Androl 25: 455-465, 2004.

18. Marcus-Braun N, Braun G, Potashnik G and Har-Vardi I: Effect of cryopreservation on quality and fertilization capacity of human sperm. Eur J Obstet Gynecol Reprod Biol 116: 63-66, 2004.

19. Agarwala A and Allamaneni SS: The effect of sperm DNA damage on assisted reproduction outcomes. A review. Minerva Ginecol 56: 235-245, 2004.

20. Zini A and Sigman M: Are tests of sperm DNA damage clinically useful? Pros and cons. J Androl 30: 219-229, 2009.

21. Elshal M, El-Sayed IH, Elsaied MA, El-Masry SA and Kumosani TA: Sperm head defects and disturbances in spermatozoal chromatin and DNA integrities in idiopathic infertile subjects: association with cigarette smoking. Clin Biochem 42: 589-594, 2009.

22. Trisini AT, Singh NP, Duty SM and Hauser R: Relationship between human semen parameters and deoxyribonucleic acid damage assessed by the neutral comet assay. Fertil Steril 82: $1623-1632,2004$

23. Rubes J, Vozdova M, Robbins WA, Rezacova O, Perreault SD and Wyrobek AJ: Stable variants of sperm aneuploidy among healthy men show associations between germinal and somatic aneuploidy. Am J Hum Genet 70: 1507-1519, 2002.

24. Matzuk MM and Lamb DJ: Genetic dissection of mammalian fertility pathways. Nat Cell Biol 4 (Suppl): S41-S49, 2002.

25. Vegetti W, Van Assche E, Frias A, et al: Correlation between semen parameters and sperm aneuploidy rates investigated by fluorescence in-situ hybridization in infertile men. Hum Reprod 15: 351-365, 2000.

26. Calogero AE, De Palma A, Grazioso C, Barone N, Romeo R, Rapazzo G and D'Agata R: Aneuploidy rate in spermatozoa of selected men with abnormal semen parameters. Hum Reprod 16: 1172-1179, 2001

27. Sharma RK, Pasqualotto AE, Nelson DR, Thomas AJ Jr and Agarwal A: Relationship between seminal white blood cell counts and oxidative stress in men treated at an infertility clinic. J Androl 22: 575-583, 2001.

28. Aitken S and Roman SD: Antioxidative system and oxidative stress in the testis. In: Molecular mechanisms in Spermatogenesis. Cheng CY (ed). Landes Bioscience, New York, 2008. 